\title{
Impact of immune checkpoint inhibitors in cancer immunotherapy.
}

\author{
Ashish Saxena and Kota V Ramana*
}

Department of Biochemistry and Molecular Biology, University of Texas Medical Branch, Galveston, USA

Accepted on 3 October, 2017

\section{Editorial}

While we are beginning to get the grip with many of the contributing factors and cellular mechanisms such as altered inflammatory, immune and oxidative stress responses, that underlie the pathophysiology of cancer growth and metastasis, and despite the development of numerous effective therapies over the past 3 to 4 decades, mortality associated with cancer remains a major health problem in the USA as well as worldwide. Although conventional cancer treatments such as radiation therapy, chemotherapy, and surgery play a significant role in controlling various primary tumours, it is not possible to completely eradicate tumour growth and spread to different organs. The practice of radiation- and chemo-therapies required special attention and based on tumour incidence and patient's metabolic process. Further, severe side effects associated with radiation and chemotherapy are one of the primary concern. Prolonged radiation therapy could also trigger normal cells to transform into neoplastic cells. At the same time, some type cancers develop resistance to chemotherapeutic drugs. Recently, the development of immunotherapies reflects a promising new approach to control cancer growth and metastasis. In the cancer immunotherapy, altering the inherent ability of the immune response to recognize and destroy cancer cells holds a firm promise towards the eradication of various types of cancer. Cancer immunotherapy is now selectively utilized for the therapy of both solid and haematological malignancies.

Cancer immunotherapies such as adoptive $\mathrm{T}$ cell transfer, oncolytic viruses, cancer vaccines, and monoclonal antibodies have diverse mechanisms of action and clinically tested in most of the cancer types. The adoptive $\mathrm{T}$ cell transfer involves the genetic modification of patient's inherent $\mathrm{T}$ cells to express receptors that specifically recognize and bind to cancer cells. Modify $\mathrm{T}$ cells are introduced back into the patient to combat cancer with long-lasting benefits. This type of approach is also referred as chimeric antigen receptor T-cell therapy and presently is in clinical trials for ovarian, leukaemia and lymphoma. Another, interesting immunotherapy technique using oncolytic viruses utilizes specially engineered viruses to infect and destroy cancer cells. These viruses specifically recognize specific antigens on the cancer cell surface and replicate and destroy them. Interestingly, when virus-infected cancer cells undergo death, they release various antigens that activate the immune system and kill more cancer cells. Currently, Talimogene laherparepvec (TVEC) is the only vaccine that is approved for melanoma treatment in the USA, Europe, and Australia. Further, SipuleucelT, a therapeutic vaccine, is available for the treatment of advanced or metastatic prostate cancer. Currently, there are more preventive vaccines available than therapeutic, including two vaccines that foster immunity against forms of the human papillomavirusassociated cancer development. Although these cancer vaccines encompass revealing the immune system to an antigen for prevention or cure, additional pre-clinical and clinical studies are required to get various oncolytic viruses and therapeutic viruses-based immunotherapies in the market. Specifically, the use of these viral-mediated immunotherapies is limited to specific cancer types, and their efficacy should also be tested in multiple cancer types.

As compared to other immunotherapies, monoclonal antibodybased therapies have been extensively investigated. Different kinds of therapies using monoclonal antibodies such as Bevacizumab (targets VEGF), Cetuximab (targets EGFR), Blinatumomab (targets both CD19 and CD3), Brentuximab (targets CD30) and Ado-trastuzumab (targets HER2) are available for the treatment of various cancers. Recently, based on the promising results of pre-clinical and clinical studies on different types of cancer, targeting of the immune checkpoint proteins to prevent tumour growth has been received significant attention in the cancer immunotherapy. Hindering the interaction between the immune checkpoint proteins such as Programmed Death 1 (PD1) and Cytotoxic T-LymphocyteAssociated protein -4 (CTLA-4) on the T- cells and PD-L1 and B7-1/B7-2 on tumour cells allows T- cells to kill tumour cells. Monoclonal antibodies against either PD-1 or PD-L1 could block the checkpoint interaction and allow T-cells to suppress the tumour growth. Similarly, antibodies against either CTLA-4 or B7-1/B7-2 could also prevent the checkpoint interaction and promote T-cell activation to kill the cancer cells. Thus, targeting the immune checkpoint proteins to enhance T-cell mediated immune responses against cancer growth is a new breakthrough for the cancer therapy. Further, FDA approval of the monoclonal antibodies against immune checkpoint proteins such as ipilimumab (targets CTLA-4), nivolumab and pembrolizumab (target PD-1) and avelumab, atezoluzumab, and durvalumab (target PD-L1), brought an exciting change in the direction of cancer immunotherapy for treating various types of cancer. Clinical trials with monoclonal antibodies targeting either PD-1 or PD-L1 have shown dramatic improvement in therapeutic response and long-term regression in patients with melanoma, bladder cancer, renal carcinoma, non-small cell lung cancer, Hodgkin's lymphoma, and skin cancer. Recent studies also suggest the potential combinational treatment with PD-1 and CTLA-4 checkpoint inhibitors have shown robust tumor regression and recommend the development of novel combinational therapy for treating the cancer patients. Anti-PD1 and anti-CTLA-4 drugs have shown the wonderful efficacy in treating ovarian cancer, renal cancer, and Hodgkin's lymphoma, even in patients who are not responsive to chemotherapy. 
Although the treatment with immune checkpoint inhibitors has brought a strong hope for cure and survival for the cancer patients, these treatments are also associated with a diverse spectrum of toxicities which could be referred as immunerelated adverse events (irAEs). These irAEs that are usually transient but could be rarely severe or even deadly. Immunemediated colitis is one of the most common side effects associated with checkpoint inhibitors such as anti-PD1 and anti-CTLA-4. Hepatitis has also been reported with immune checkpoint inhibitors. In addition to this, immune-mediated pneumonitis that affects lungs may also occur at any point during and after treatment with checkpoint inhibitors specifically with anti-CTLA-4 inhibitors. Furthermore, some cases of immune-mediated cutaneous toxicities such as vitiligo, Stevens-Johnson syndrome, Sweet's syndrome, toxic epidermal necrolysis, bullous pemphigoid, and lichen sclerosis have also been reported. The incidence of thyroid disorders are also reported for both anti-CTLA4 and antiPD1 monotherapy, but the complication increases when given as a combination therapy with both the antibodies. Further, recent studies also suggest that some cancer patients become resistant to checkpoint inhibitors. Therefore, more randomized clinical studies are required to understand how immune checkpoint inhibitors increase the unwanted inflammatory and immune burst and cause - toxicities. Use of various combinational therapies that can improve the efficacy of immune checkpoint inhibitors as well as reduce the immune-mediated side effects is necessary to treat cancer patients successfully. Without a doubt, the immune checkpoint inhibitors have recently provided significant advances in the care of individuals with a variety of advanced solid tumours. Further investigations using various pre-clinical animal studies and patient-derived clinical samples are required to identify the fundamentals of cancer immune regulation and its effect on tumour growth, metastasis, and acquired drug resistance. Identification of novel immune checkpoint proteins and receptors and demonstration of the mechanisms of action will not only increase the diversity of cancer immunotherapy but also improve patient's safety. The additional pre-clinical trials with combinational treatments could create a new hope for patients affected not only by cancer but also by other types of disease such as diabetes and cardiovascular complications. However, a better approach using combinational therapies using monoclonal antibodies with non-steroidal anti-inflammatory agents, potent antioxidants and small molecular inhibitors could also be tested to enhance the efficacy of immune checkpoint inhibitors as well as to reduce the immune-mediated side effects.

\section{*Correspondence to}

Kota V Ramana

Department of Biochemistry and Molecular Biology

University of Texas Medical Branch,

USA

E-mail: kvramana@utmb.edu 